\title{
Maximum Likelihood Detection with Intermittent Observations
}

\author{
Ashraf Tantawy, Student Member, IEEE, Xenofon Koutsoukos, Senior Member, IEEE, \\ and Gautam Biswas, Senior Member, IEEE
}

\begin{abstract}
The decentralized detection performance, using wireless passive sensor networks, is analyzed according to the minimum probability of error criterion. Passive sensors communicate their measurements to the reader using data network packets, and therefore, the two main phenomena affecting the detection performance are packet loss and packet delay. In this paper, we formulate the decentralized detection problem with passive sensors and show that the optimal decision rule with packet loss is the likelihood ratio test. We present a comparative analysis study between detection with ideal and non-ideal channels, for the problem of DC level detection in White Gaussian Noise. We validate the analytical results using Monte Carlo Simulation study. Finally, we present a simple scheme for adaptive detector design, to restore the original detection performance, with the cost of increasing the delay for detection.
\end{abstract}

\section{INTRODUCTION}

Decentralized Detection (DD) has been an active area of research in the last two decades. In DD, a group of nodes sense the environment, and collaborate with each other to reach a final decision about the state of nature. Different architectures have been proposed for the communication between nodes. In a fully distributed architecture, nodes communicate with each other to reach a final decision, with no fusion center. On the other hand, in the serial architecture, nodes are arranged in tandem, and every node passes its decision to the following node, with the last node taking the final decision. In the decentralized architecture, nodes communicate only with a central location, called the fusion center, which analyzes the information and makes the final decision [18]. The decentralized architecture has attracted considerable attention in the research community due to its practical significance and analytic tractability. The main problem in decentralized detection is to design both the optimal local decision rules and the fusion rule to detect events as accurately as possible [2].

Emergence of wireless sensor networks has produced a new paradigm for decentralized detection. The main difference comes from the fact that wireless sensors communicate their measurements to the fusion center over an unreliable communication channel, and therefore, perfect information is no longer available at the fusion center. This fact was overlooked in early developments of the field.

In the last few years, passive wireless sensors have started to emerge as a replacement technology for powered sensors

The authors are with the Institute for Software Integrated Systems and the Department of Electrical Engineering and Computer Science, Vanderbilt University, Nashville, TN, 37235, USA (email: ashraf.tantawy@vanderbilt.edu; xenofon.koutsoukos@vanderbilt.edu; gautam.biswas@vanderbilt.edu). in many different fields. The main attraction for passive sensors is that they do not need any power source. The basic idea is to use a powered reader to poll the data from the different passive sensors. A passive sensor uses the incident power from the reader to energize its local circuitry. A variety of ways exist for the sensor to modulate the reader incident wave and relay its measurement back to the reader. The most popular example for this architecture is RFID technology [8].

Motivated by RFID technology, various sensors have been integrated with RFID tags [14]. In this integrated architecture, passive sensors communicate arbitrary sensor data by emulating an RFID tag. The tag ID encodes the desired sensor data, and through a specially-designed software in the reader, sensor data submitted in the payload of the RFID tag data packet can be decoded. This integration adheres to RFID industry standards, and therefore, it is compatible with a variety of RFID readers.

The decentralized detection problem, using wireless passive sensor networks, is different from the classical decentralized detection problem in several different aspects. Passive sensors communicate their measurements to the reader using data network packets, adhering to strict communication protocol standards. Accordingly, the effect of the communication channel on the received waveforms is of interest only to the detection task performed by the communication system at the physical layer level, to infer the transmitted symbol (representing a string of bits). As far as the sensor data (in the packet payload) is concerned, the data may not arrive at all, or it may arrive with arbitrary delay.

Another property of passive sensors is that they lack sufficient computational power to preprocess measurements before they are submitted to the fusion center. Therefore, we have to assume that either the raw measurements are submitted directly, or a very limited preprocessing takes place at the node. The preprocessing time is limited by the amount of time that the reader supplies power to the sensors, and this is the polling period. Also, the reader polls different sensors at the same time over a shared communication media, which can give rise to collisions, packet loss, and packet delay. Finally, the reflected signal from passive sensors are very weak, due to regulatory laws for RFID power, and hence the detection task becomes much more difficult since the signal to noise ratio is not completely under the designer's control.

In previous work, we studied the degradation in the detector performance as a result of channel imperfection [15], using the Neyman-Pearson (NP) formulation. We assumed a single passive sensor to reader communication 
over a Bernoulli channel, to model the missing observations effect of a typical data network. We obtained the detector performance analytically and by Monte Carlo simulations, and proposed a heuristic approach to restore the detector performance, by increasing the number of samples, and hence increasing the detection delay.

In this paper, we apply the Maximum Likelihood (ML) criterion to study the detection performance in wireless passive sensors. This is a special case of the minimum probability of error criterion. We formulate the detection problem and derive the optimal fusion rule with a nonideal communication channel between the reader and passive sensors. We present a comparative analysis between detector performance with ideal and non-ideal channels.

The rest of the paper is organized as follows: In Section III, we formulate the detection problem using wireless passive sensors. Section IV presents the classical results of detection performance with minimum probability of error criterion. In Section V, we derive the optimal decision rule for the detector with packet loss. We present a comparative analysis, using a DC level detection problem as a case study. In Section VII, we propose a simple adaptive architecture to restore the original detector performance, Finally, we conclude the work with future research directions in section VIII.

\section{RELATED WORK}

The research on decentralized detection is largely attributed to the seminal work of Tenney and Sandell [16]. The optimal decision rules for the local nodes and the fusion center are derived under various problem settings and different optimality criteria. Optimal data fusion rule is derived in [1]. The optimality of the likelihood ratio for local nodes decision is proved in [18]. Detection performance with node failures is studied in [13]. For a more comprehensive survey in this area, the readers are referred to [2], [4] and the references therein. Decision fusion with network delays and channel errors has been considered in [17].

For DD with WSN, different constraints are studied and included in the design process. Channel-aware decision fusion for fading channels, given fixed local decision rules, is studied in [3], with the problem revisited in [12] to relax the assumption of channel state information availability at the fusion center. Distributed detection with channel errors is studied in [10], assuming Binary Symmetric Channel. The optimality of the Likelihood Ratio (LR) test for local sensor decisions, with a non ideal channel, is proved in [5]. The detection and estimation performances, when the sensors and the fusion center communicate over multi-access fading channel are studied in [11], where it is assumed that the sensor transmits to the fusion center the type of the sensor observations. The design of the optimal quantizer and fusion rule, using both Bayesian and Neyman-Pearson approaches, when the transmission is subject to noise and inter-channel interference is discussed in [6]. The distributed detection problem over Multiple Access Channel (MAC), as opposed to the traditional assumption of parallel access channel, is studied in [9].
The performance analysis for NP detectors, with nonideal channel, is studied in [15]. A Bernoulli communication channel is assumed, and closed form expressions are derived for the performance metrics provided a sufficient statistic exists for the data samples. The analysis is augmented by Monte Carlo Simulation studies, and an adaptive detector design is proposed, with the penalty of increasing the delay for detection.

\section{PROBLEM Formulation}

Figure 1 illustrates the detection system architecture. We consider the problem of signal detection using a collection of $l$ passive sensors, measuring the same phenomenon, and polled by a common reader. Passive sensors have no power source, and are awakened by the polling signal of the reader, to submit their information. We assume the passive sensor has limited computational power, and since it is active only during the polling cycle, we assume it samples the sensed signal, time-stamps the raw observation, and submits it without preprocessing, to the reader. We further assume that the underlying communication protocol handles the synchronization between the reader and the passive sensors.

For each polling cycle, every passive sensor submits its measurement over the shared multi-access channel. Since the communication channel is shared, collisions are likely. We assume that the data from the colliding sensors is lost. We do not assume that collided packets will be retransmitted for two reasons; (1) the passive sensor has to have built in memory to store the previously-submitted information, which it has to retransmit again in case of a collision, and (2) outdated data may not be useful.

At the end of every polling cycle, the reader collects the observations and arranges them chronologically, using their time-stamps. The reader takes a decision based on the collected data vector, $\mathbf{x}$, regardless of the number of observations received. This is required in detection systems, since the delay of detection is one of the performance metrics. We designate the polling cycle time by $T_{p}$. The reader in this system architecture has the additional role of the data fusion center in the classical decentralized detection approach.

We use a probabilistic model for the communication channel. At the end of the polling cycle time, $T_{p}$, there are a number of observations received, viz. $K$. This is a random variable, characterized by its Probability Mass Function $(\mathrm{PMF}) p_{K}(k)$. Since any practical channel has a finite capacity, the maximum value of the realization of $K$ has an upper bound $N$. The lower bound is 0 , which represents the case of receiving no observations during a time period $T_{p}$. The latter case is not expected for any useful practical channel.

In the ideal channel case the reader has complete information about the measurements. Therefore, we expect a degradation in the detection performance. The work in this paper quantifies the performance loss, and attempts to recover the original detector performance. 


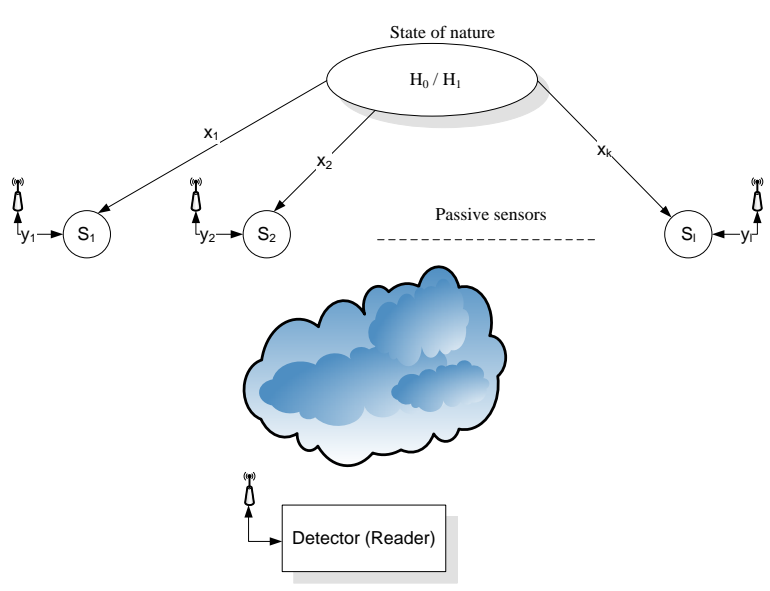

Fig. 1. Detection with passive sensors

\section{PRELIMINARIES}

We consider the binary hypothesis testing problem where the state of nature is represented by one of two hypotheses, $\mathcal{H}_{0}$ and $\mathcal{H}_{1}$, with prior probabilities $P\left(\mathcal{H}_{0}\right)$ and $P\left(\mathcal{H}_{1}\right)$, respectively. An observation vector, $\mathbf{x}$, is used to statistically validate one of the two hypotheses. The decision is governed by the minimum probability of error criterion, for minimizing the average probability of error, $P_{e}$. The probability of error is defined as:

$$
P_{e}=P\left[\mathcal{H}_{i} \mid \mathcal{H}_{j}\right] \quad i, j \in\{0,1\}, i \neq j
$$

and the average probability of error is given by:

$$
E\left[P_{e}\right]=P\left[\mathcal{H}_{1} \mid \mathcal{H}_{0}\right] P\left[\mathcal{H}_{0}\right]+P\left[\mathcal{H}_{0} \mid \mathcal{H}_{1}\right] P\left[\mathcal{H}_{1}\right]
$$

The optimal test to minimize the probability of error is given by:

$$
\frac{P\left[\mathbf{x} \mid \mathcal{H}_{1}\right]}{P\left[\mathbf{x} \mid \mathcal{H}_{0}\right]} \underset{\mathcal{H}_{0}}{\stackrel{\mathcal{H}_{1}}{\gtrless}} \frac{P\left[\mathcal{H}_{0}\right]}{P\left[\mathcal{H}_{1}\right]}
$$

When prior probabilities are equal, the test reduces to deciding the hypothesis with the larger conditional likelihood, $P\left(\mathbf{x} \mid \mathcal{H}_{i}\right), i \in\{0,1\}$.

Since the hypotheses $\mathcal{H}_{0}$ and $\mathcal{H}_{1}$ are random variables, the probability of error, $P_{e}$, is also a random variable with two possible outcomes, $P_{m}=P\left(\mathcal{H}_{0} \mid \mathcal{H}_{1}\right)$ and $P_{f}=P\left(\mathcal{H}_{1} \mid \mathcal{H}_{0}\right)$, and probabilities $P\left(\mathcal{H}_{1}\right)$ and $P\left(\mathcal{H}_{0}\right)$, respectively. The random variable $P_{e}($.$) is defined over the sample space \Omega_{\mathcal{H}}=$ $\left\{\mathcal{H}_{0}, \mathcal{H}_{1}\right\}$. This viewpoint becomes useful when extended to include the probabilistic channel effect, in Section V.

\section{OPTIMAL DECISION RULE}

The introduction of the probabilistic channel model between the reader and passive sensors changes the sample space over which the random variable $P_{e}$ is defined. If we define the sample space for the random variable $K$, representing the number of samples received in a time period
$T_{p}$, by $\Omega_{K}=\{0,1 \cdots N\}$, then the new sample space for $P_{e}$ is given by:

$$
\Omega_{p_{e}}=\Omega_{\mathcal{H}} \times \Omega_{K}=\left\{\left(\mathcal{H}_{0}, 0\right),\left(\mathcal{H}_{0}, 1\right) \cdots,\left(\mathcal{H}_{1}, N\right)\right\}
$$

and the probability of error is defined as:

$$
P_{e}=P\left[\mathcal{H}_{i} \mid \mathcal{H}_{j}, k\right] \quad i, j \in\{0,1\}, k \in\{0,1 \cdots, N\}
$$

and the average probability of error is given by:

$$
\begin{aligned}
E\left[P_{e}\right] & =\sum_{\substack{i, j=0 \\
i \neq j}}^{1} \sum_{K=1}^{N} P\left[\mathcal{H}_{i} \mid \mathcal{H}_{j}, K\right] P\left[\mathcal{H}_{j}, K\right] \\
& =\sum_{\substack{i, j=0 \\
i \neq j}}^{1} \sum_{K=1}^{N} P\left[\mathcal{H}_{i} \mid \mathcal{H}_{j}, K\right] P\left[\mathcal{H}_{j}\right] P[K]
\end{aligned}
$$

since the hypotheses $\mathcal{H}_{i}, i \in\{0,1\}$, and the number of observations $K$ are independent. If the prior probabilities are equal, i.e. $P\left[\mathcal{H}_{0}\right]=P\left[\mathcal{H}_{1}\right]=\frac{1}{2}$, then the average probability of error is given by:

$$
E\left[P_{e}\right]=\frac{1}{2} \sum_{\substack{i, j=0 \\ i \neq j}}^{1} \sum_{K=1}^{N} P\left[\mathcal{H}_{i} \mid \mathcal{H}_{j}, K\right] P[K]
$$

The optimal decision rule that minimizes $E\left[P_{e}\right]$, in Equation (4), could be derived as follows:

$$
\begin{aligned}
E\left[P_{e}\right] & =\sum_{k=1}^{N} P[k]\left(P\left[\mathcal{H}_{0} \mid \mathcal{H}_{1}, k\right] P\left[\mathcal{H}_{1}\right]+P\left[\mathcal{H}_{1} \mid \mathcal{H}_{0}, k\right] P\left[\mathcal{H}_{0}\right]\right) \\
& =\sum_{k=1}^{N} P[k]\left[\int_{R_{0}^{k}} P\left[\mathbf{x} \mid \mathcal{H}_{1}, k\right] P\left[\mathcal{H}_{1}\right]+\int_{R_{1}^{k}} P\left[\mathbf{x} \mid \mathcal{H}_{0}, k\right] P\left[\mathcal{H}_{0}\right]\right] \\
& =\sum_{k=1}^{N} P[k] \int_{R_{0}^{k}}\left(P\left[\mathbf{x} \mid \mathcal{H}_{1}, k\right] P\left[\mathcal{H}_{1}\right]-P\left[\mathbf{x} \mid \mathcal{H}_{0}, k\right] P\left[\mathcal{H}_{0}\right]\right)
\end{aligned}
$$

where $R_{0}^{k}$ and $R_{1}^{k}$ represent the decision regions for hypothesis $\mathcal{H}_{0}$ and $\mathcal{H}_{1}$, respectively, when the number of samples received at the reader is equal to $k$. The average probability of error is minimized by including $\mathbf{x}$ in $R_{0}^{k}$ when the quantity between brackets is negative:

$$
P\left[\mathbf{x} \mid \mathcal{H}_{1}, k\right] P\left[\mathcal{H}_{1}\right]-P\left[\mathbf{x} \mid \mathcal{H}_{0}, k\right] P\left[\mathcal{H}_{0}\right]<0
$$

Equivalently, the decision rule is given by:

$$
\frac{P\left[\mathbf{x} \mid \mathcal{H}_{1}, k\right]}{P\left[\mathbf{x} \mid \mathcal{H}_{0}, k\right]} \underset{\mathcal{H}_{0}}{\stackrel{\mathcal{H}_{1}}{\gtrless}} \frac{P\left[\mathcal{H}_{0}\right]}{P\left[\mathcal{H}_{1}\right]}
$$

Hence, the optimal decision rule with missing observations is the likelihood ratio test.

Section VI presents an example detector and illustrates the difference between the performance with the ideal channel, given by Equation (2), and the performance with the nonideal channel, given by Equation (5). 


\section{ML DETECTION-DC LEVEL IN WGN}

In this section, we consider the problem of detecting a known DC level in White Gaussian Noise (WGN), using the passive sensor network architecture illustrated in Figure 1. The problem is formulated as:

$$
\begin{array}{ll}
\mathcal{H}_{0} & : \quad x[n]=\omega[n] \\
\mathcal{H}_{1} & : \quad x[n]=A+\omega[n]
\end{array}
$$

where $n=0,1, \ldots, N-1, x[n]$ represents the sample sequence, $A$ is the known DC level to be detected, and $w[n]$ represents WGN with zero mean and variance $\sigma^{2}$.

\section{A. Probability of Error Distribution}

In case of WGN, it can be shown that the test statistic reduces to the sample mean, and therefore, $P_{e}$ is given by [7]:

$$
P_{e}=Q\left(\sqrt{\frac{N A^{2}}{4 \sigma^{2}}}\right) \quad \mathcal{H}_{0}, \mathcal{H}_{1}
$$

where $Q($.$) is the error function defined by:$

$$
Q(x)=\int_{x}^{\infty} \frac{1}{\sqrt{2 \pi}} e^{-\frac{1}{2} t^{2}} d t
$$

Now we assume a communication channel modeled as an IID Bernoulli random process, with a probability of missing an observation $\lambda$. The total number of observations received at the reader for the Bernoulli channel is given by:

$$
P[K=k]=\left(\begin{array}{c}
N \\
k
\end{array}\right)(1-\lambda)^{k} \lambda^{(N-k)}
$$

To use Equation (3), we note that the optimal decision rule with packet loss (Equation (6)) results in the same test statistic, the sample mean. Accordingly:

$$
P\left[\mathcal{H}_{0} \mid \mathcal{H}_{1}, k\right]=P\left[\mathcal{H}_{1} \mid \mathcal{H}_{0}, k\right]=Q\left(\frac{A / 2}{\sqrt{\sigma^{2} / k}}\right)
$$

and the probability of error, $P_{e}$, is given by:

$$
P_{e}=Q\left(\sqrt{\frac{K A^{2}}{4 \sigma^{2}}}\right) \quad \mathcal{H}_{i}, K
$$

Figure 2 illustrates the PMF for the random variable $P_{e}$ with ideal channel (Equation (7)), and Bernoulli channel (Equation 10), for the parameters in Table I. Since the probabilities are symmetric under $\mathcal{H}_{0}$ and $\mathcal{H}_{1}$, only one case is shown. It is clear how the distribution of $P_{e}$ spreads over with non-ideal channel, and therefore, $E\left[P_{e}\right]$ for the non-ideal channel case is anticipated to be higher, and accordingly, the detector performance is degraded. This fact is confirmed and quantified in the next section.

TABLE I

DETECTOR PARAMETERS

\begin{tabular}{|c||c|c|c|c|}
\hline Parameter & $A$ & $\sigma$ & $N$ & $\lambda$ \\
\hline Value & 0.2 & 1 & 50 & 0.2 \\
\hline
\end{tabular}

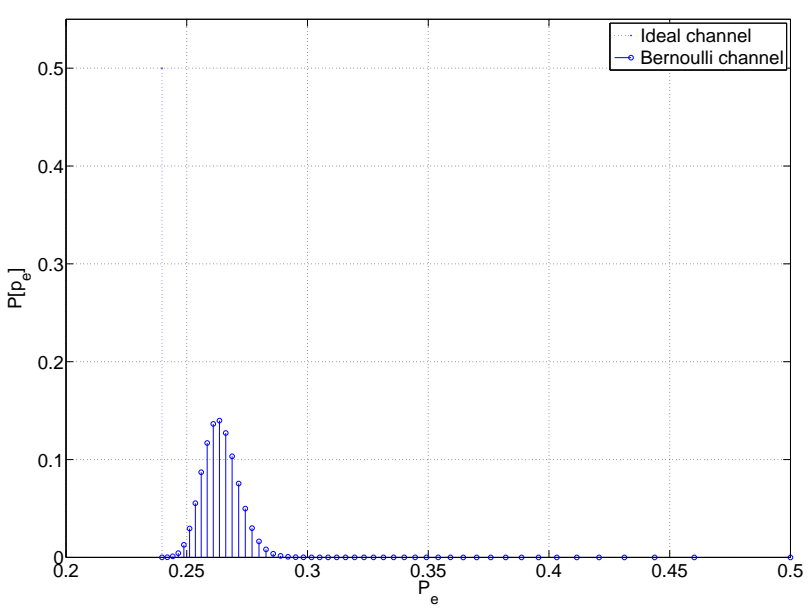

Fig. 2. Probability Mass Function for $P_{e}$, ideal versus Bernoulli channel

\section{B. DETECTION PERFORMANCE}

The expected value of $P_{e}$ with ideal channel is obtained by applying Equation (7) to Equation (2):

$$
E\left[P_{e}\right]=Q\left(\sqrt{\frac{N A^{2}}{4 \sigma^{2}}}\right)
$$

The expected value of $P_{e}$ with Bernoulli channel is obtained by applying Equation (10) to Equation (5):

$$
E\left[P_{e}\right]=\sum_{k=1}^{N}\left(\begin{array}{l}
N \\
k
\end{array}\right)(1-\lambda)^{k} \lambda^{(N-k)} Q\left(\sqrt{\frac{k A^{2}}{4 \sigma^{2}}}\right)
$$

We note that equation (12) reduces to equation (11) when $\lambda=0$.

Example 1. Assume we have an ML detector with the parameters shown in Table I. With an ideal channel, we use Equation (11) to get $E\left[P_{e}\right]=0.2398$. With the Bernoulli channel, and packet drop rate $\lambda=0.2$, and using Equation (12), we get $E\left[P_{e}\right]=0.2637$. The degradation in the performance is best illustrated by the error probability curve, as explained in the next section.

\section{ERROR PROBABILITY CURVE}

Figure 3 plots Equation (12), for different values of $\lambda$, including the ideal case $(\lambda=0)$, using the parameters in Table I. The performance degradation is clear, where $E\left[P_{e}\right]$ increases, for the same number of samples, with increasing $\lambda$.

To verify the analytical results, Monte Carlo simulation experiments are conducted. Samples are generated from two different Gaussian distributions (corresponding to the two hypotheses), with equal probability. A Bernoulli channel is introduced in the signal path to the detector. The detector calculates a running average, and it ignores dropped observations. The detector compares the running average to a threshold value $A / 2$. The error occurs if the running average 


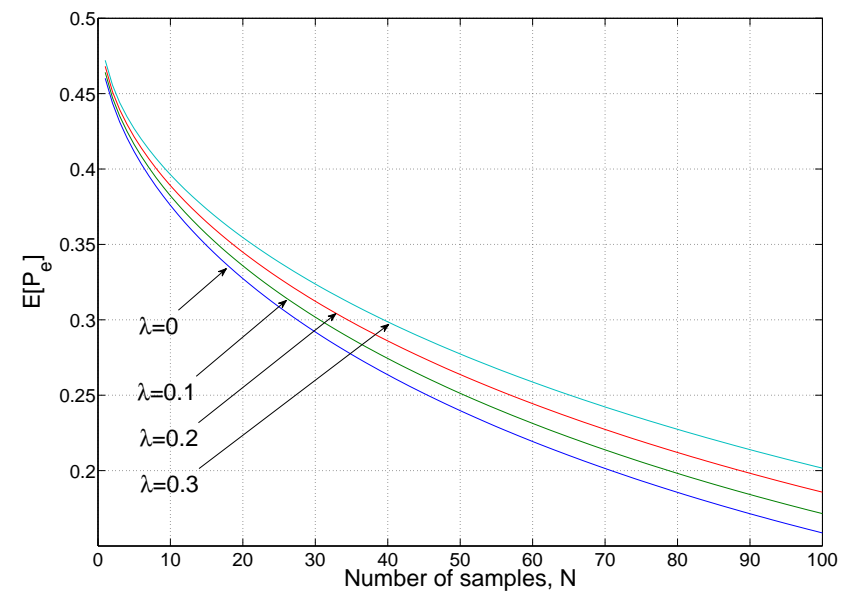

Fig. 3. $E\left[P_{e}\right]$ for different channel drop rate, $\lambda$

exceeds $A / 2$ and the true hypothesis is $\mathcal{H}_{0}$, or if the running average is less than $A / 2$ and the true hypothesis is $\mathcal{H}_{1}$. For every value of the number of samples for the detector, $N$, 10,000 Monte Carlo trials are performed to get an accurate value for $E\left[P_{e}\right]$. Table II lists the parameters used in the simulation experiment.

TABLE II

Monte CARlo Simulation Parameters

\begin{tabular}{|c||c|c|c|c|}
\hline Parameter & $A$ & $\sigma$ & $N$ & $\lambda$ \\
\hline Value & $0,0.2$ & 1 & $(5: 100)$ & $0,0.3$ \\
\hline
\end{tabular}

Figure 4 shows the theoretical $E\left[P_{e}\right]-N$ curve (Equation (12)) versus the curve obtained from Monte Carlo simulation, for $\lambda=0$ and $\lambda=0.3$. As illustrated in the figure, the two curves for each value of $\lambda$ are very similar. The minor difference is a result of Monte Carlo simulation accuracy. Asymptotically (MC trials $\rightarrow \infty$ ), the two curves for each value of $\lambda$ coincide.

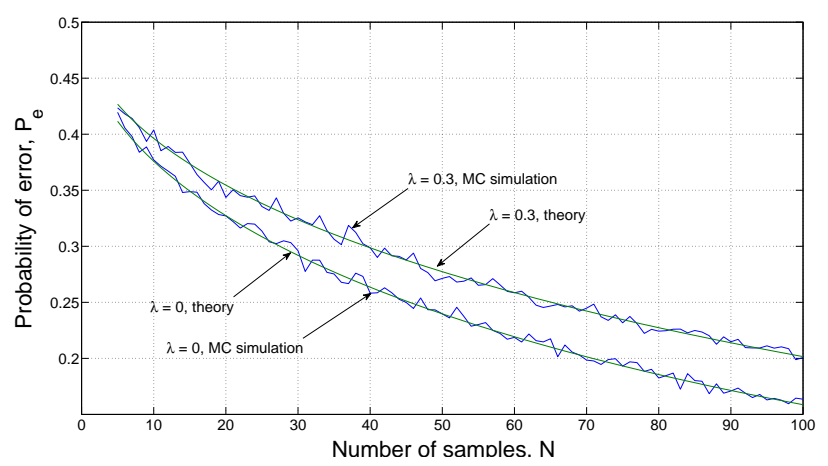

Fig. 4. $E\left[P_{e}\right]$ against number of samples $N$, Monte Carlo simulations

\section{ADAPTIVE DETECTOR DESIGN}

To compensate for the degradation in the performance of the detector, we need to equate the expressions for $E\left[P_{e}\right]$ before and after the degradation:

$$
\begin{array}{r}
P\left[\mathcal{H}_{1} \mid \mathcal{H}_{0}\right] P\left[\mathcal{H}_{0}\right]+P\left[\mathcal{H}_{0} \mid \mathcal{H}_{1}\right] P\left[\mathcal{H}_{1}\right]= \\
\sum_{k=1}^{N} P[k]\left(P\left[\mathcal{H}_{1} \mid \mathcal{H}_{0}, k\right] P\left[\mathcal{H}_{0}\right]+P\left[\mathcal{H}_{0} \mid \mathcal{H}_{1}, k\right] P\left[\mathcal{H}_{1}\right]\right)
\end{array}
$$

The degrees of freedom for Equation (13) are the upper bound for the number of samples, $N$, and the channel probabilistic distribution $P[K]$. The channel distribution could be controlled by modifying $N$, as can be easily shown in the Bernoulli channel example discussed in section V. Increasing $N$ can be achieved in one of two ways: (1) increasing the channel capacity, and (2) increasing the reader polling cycle time, $T_{p}$. While the first option has no impact on the detector performance, the latter adds to the delay for detection.

For the DC level detection problem, discussed in section VI, we need to find the minimum value for $N$ that restores the original detector performance $E\left[P_{e}\right]=\alpha$ :

$$
\begin{aligned}
& \min _{E\left[P_{e}\right]} N \\
& \sum_{k=1}^{N}\left(\begin{array}{l}
N \\
k
\end{array}\right)(1-\lambda)^{k} \lambda^{(N-k)} Q\left(\sqrt{\frac{k A^{2}}{4 \sigma^{2}}}\right)-E\left[P_{e}\right]=0 \\
& \text { subject to } E\left[P_{e}\right]=\alpha
\end{aligned}
$$

Since $N$ is a monotonically decreasing function of $E\left[P_{e}\right]$, as Figure 3 illustrates, we do not need to solve the optimization problem. We can obtain the value of $N$ by directly solving the following equation numerically:

$$
\sum_{k=1}^{M}\left(\begin{array}{c}
M \\
k
\end{array}\right)(1-\lambda)^{k} \lambda^{(M-k)} Q\left(\sqrt{\frac{k A^{2}}{4 \sigma^{2}}}\right)-\alpha=0
$$

where $N=\lceil M\rceil$

By solving Equation (14) for $N$, given different values of the channel drop rate, $\lambda$, we get an $N-\lambda$ relationship, where the channel drop rate, $\lambda$, is plotted on the abscissa $(0 \leq$ $\lambda \leq 1)$ and the corresponding number of samples required to restore the performance, $\tilde{N}$, is plotted on the ordinate. Figure 5 illustrates the $N-\lambda$ curve for the example detector given in this paper, with parameters as in Table I. It is shown that the number of samples approximately doubles with a drop rate $\lambda=0.5$, and the curve exhibits an exponential behavior thereafter.

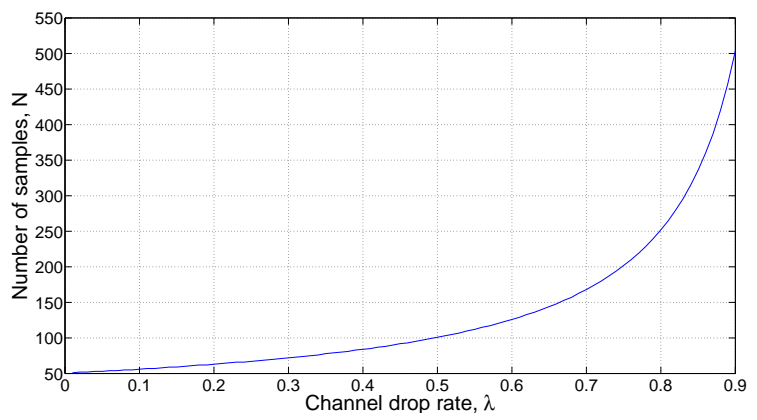

Fig. 5. Optimal number of samples with channel drop rate 
The curve in Figure 5 can be used to estimate the number of samples required to achieve a specific performance measure, under different channel conditions. If the channel statistical parameters can be estimated online, then the curve can be used to adapt the detector online, by changing the number of samples $N$ according to the current channel drop rate. As mentioned before, $N$ could increase by either increasing the channel capacity, or adding more passive sensors.

\section{CONCLUSIONS AND FUTURE WORK}

In this paper we have formulated the decentralized detection problem, using wireless passive sensors. We used the practical fact that wireless sensors communicate measurements using data network packets, and therefore, we have studied the effect of dropped packets on the detection performance. We have shown that the optimal decision rule, according to the minimum probability of error criterion, reduces to the likelihood ratio test, when we account for the non-ideal channel between the reader and the passive sensors. We have presented a comparative analysis between the detection performance with ideal and non-ideal channels, using the problem of DC level detection in WGN. We have quantified the loss in the detector performance, and presented a simple adaptive method to restore the original detector performance, with the drawback of increasing the delay for detection.

In this paper, we have also studied the effect of packet drop on the detection performance. Packet delay is another phenomenon in data networks that affects the detection process. This is currently under study. Also, in the adaptive algorithm described in this paper, we assumed that increasing the number of samples has no effect on the channel parameters. In wireless passive sensor networks, increasing the number of samples is achieved by adding more sensors, which causes an increase in the collision rate, and hence changes in the probabilistic channel model. We are currently studying this problem in more detail.

\section{ACKNOWLEDGMENT}

This work was supported in part by the National Science Foundation under Grant CNS-06152140, and NASA NRA NNX07AD12A.

\section{REFERENCES}

[1] Z. Chair and P. Varshney, "Optimal data fusion in multiple sensor detection systems," Aerospace and Electronic Systems, IEEE Transactions on, vol. AES-22, no. 1, pp. 98-101, Jan. 1986.

[2] J.-F. Chamberland and V. V. Veeravalli, "Wireless sensors in distributed detection applications," IEEE Signal Processing Magazine, 2007.

[3] B. Chen, R. Jiang, T. Kasetkasem, and P. K. Varshney, "Channel aware decision fusion in wireless sensor networks," IEEE Transactions on Signal Processing, vol. 52, no. 12, pp. 3454-3458, Dec. 2004.

[4] B. Chen, L. Tong, and P. K. Varshney, "Channel-aware distributed detection in wireless sensor networks," IEEE Signal Processing Magazine, July 2006.

[5] B. Chen and P. K. Willett, "On the optimality of the likelihoodratio test for local sensor decision rules in the presence of nonideal channels," IEEE Transactions on Information Theory, vol. 51, no. 2, pp. 693-699, Feb. 2005.

[6] T. M. Duman and M. Salehi, "Decentralized detection over multipleaccess channels," IEEE Transactions on Aerospace and Electronic Systems, vol. 34, no. 2, pp. 469-476, Apr 1998.

[7] S. M. Kay, Fundamentals of Statistical Signal Processing, Volume 2: Detection Theory, ser. Prentice Hall Signal Processing Series, A. V. Oppenheim, Ed. Prentice Hall PTR, 1998.

[8] S. Lewis, "A basic introduction to RFID technology and its use in the supply chain," LARAN RFID, Tech. Rep., 2004.

[9] W. Li and H. Dai, "Distributed detection of a deterministic signal in correlated gaussian noise over mac," IEEE International Symposium on Information Theory, pp. 2134-2138, July 2006.

[10] M. Madishetty, V. Kanchumarthy, R. Viswanathan, and C. Gowda, "Distributed detection with channel errors," Proceedings of the ThirtySeventh Southeastern Symposium on System Theory, SSST '05., pp. 302-306, March 2005.

[11] G. Mergen and L. Tong, "Sensor-fusion center communication over multiaccess fading channels," IEEE International Conference on Acoustics, Speech, and Signal Processing, ICASSP 05, vol. 4, pp. iv/841-iv/844 Vol. 4, March 2005.

[12] R. Niu, B. Chen, and P. Varshney, "Fusion of decisions transmitted over rayleigh fading channels in wireless sensor networks," IEEE Transactions on Signal Processing, vol. 54, no. 3, pp. 1018-1027, March 2006.

[13] A. Reibman and L. Nolte, "Optimal design and performance of distributed signal detection systems with faults," IEEE Transactions on Acoustics, Speech and Signal Processing, vol. 38, no. 10, pp. 17711782, Oct 1990.

[14] A. P. Sample, D. J. Yeager, P. S. Powledge, and J. R. Smith, "Design of a passively-powered, programmable sensing platform for UHF RFID systems," in IEEE International Conference on RFID, 2007.

[15] A. Tantawy, X. Koutsoukos, and G. Biswas, "Detection using intermittent observations for passive wireless sensors," American Control Conference, ACC2009, 2009.

[16] R. R. Tenney and N. R. Sandell, "Detection with distributed sensors," IEEE Transactions on Aerospace and Electronic Systems, vol. AES17, no. 4, pp. 501-510, July 1981.

[17] S. C. A. Thomopoulos and L. Zhang, "Distributed decision fusion with networking delays and channel errors," Inform. Sci., vol. 66, pp. 91-118, 1992.

[18] J. N. Tsitsiklis and M. Athans, "On the complexity of decentralized decision making and detection problems," Decision and Control, 1984. The 23rd IEEE Conference on, vol. 23, pp. 1638-1641, Dec. 1984. 